\title{
FORMEN DER REGELUNG DES STAATS- ANGEHÖRIGKEITSRECHTS IN DEN 141 STAATEN DER WELT
}

\author{
Von Hellmuth Hecker
}

Im Augenblick gibt es 141 Staaten, die Souveränität als Völkerrechtssubjekte in Anspruch nehmen, wobei Biafra als Bürgerkriegsgebiet nicht mitgezählt ist. Mit dem Entstehen dieser 141 Staaten entstand auch eine Staatsangehörigkeit, denn zu den Wesensmerkmalen des soziologischen Phänomens "Staat" gehören auch die Bewohner des betreffenden Gebietes, eben die Untertanen, Staatsbürger oder Staatsangehörigen. Welche Personen der jeweilige Staat als seine Gewaltunterworfenen in Anspruch nimmt und wie lange er dies tut, ist eine Frage, die unbedingt gelöst werden muß. Die Gründe für Erwerb und Verlust der StA und die dazugehörigen Verfahrensvorschriften sind ein wesentlicher Bestandteil des öffentlichen Rechts eines Staates, und zwar des Staatsrechts oder des Verfassungsrechts im weiteren Sinne, das erheblich über die geschriebene Verfassungsurkunde hinausgehen kann. Die Formen der Regelung des Staatsangehörigkeitsrechts lassen sich nach wenigen Grundtypen unterscheiden. Es gibt eine Regelung in der Verfassung, in Verfassung und Gesetz, im BGB, in einem Staatsvertrag, und fünftens in einem besonderen Staatsangehörigkeitsgesetz. Als sechste Möglichkeit kommt hinzu, daß ein Staat das StA-Recht dem Gewohnheitsrecht überläßt. Diese sechs Formen der Regelung verteilen sich zur Zeit wie folgt auf die 141 Staaten der Welt:

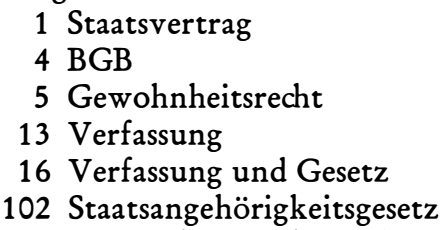

Dazu ist im einzelnen zu bemerken:

1. Der zur Zeit einzige Staat, dessen StA in einem völkerrechtlichen Vertrag grundlegend geregelt ist, ist Cypern. Im Vertrag zwischen Großbritannien, Griechenland und der Türkei vom 16. 8. 1960 findet sich im Annex D eine ausführliche Regelung der Erwerbs- und Verlustgründe der StA.

2. Die Zahl der Staaten, die die StA im BGB regeln, ist im Absterben. Diese Form, die auf den Code Civil Napoleons von 1804 zurückgeht und die Frankreich selber schon 1927 verlassen hat, findet sich nur noch in Staaten, die irgendwie unter dem Einfluß des französischen Rechts, des romanischen Rechtskreises, standen. Da ist zunächst Monaco, das die StA im BGB von 1818 geregelt hat, wobei aber nicht eine der heute geltenden Fassungen der betreffenden Artikel unverändert geblieben ist. Ferner gab Frankreich seinem Protektorat Kambodscha 1915 ein BGB, dessen StAB'en bis heute gelten, lediglich 1954 durch ein EBG ergänzt. Spanien ging erst 1889 dazu über, die bisher in den Verfassungen geregelte StA im BGB zu behandeln, dessen StAB'en 1954 neu gefaßt wurden, während der Entwurf eines eigenen StAG von 1941 nie Gesetz wurde. Als vierter Staat hat der Iran die StA seit 1935 im BGB geregelt. Alle vier Staaten dieser Gruppe sind Monarchien.

3. Am schwierigsten ist das StA-Recht in den fünf Staaten zu bestimmen, die keine geschriebene Regelung besitzen, nämlich: in Europa San Marino und in Asien vier Staaten (China, Maskat, Mongolei, Yemen). In jedem Staat liegen besondere historisch-politische Gründe dafür vor, daß es dort keine StA-Vorschriften gibt, Gründe, deren nähere Behandlung in diesem Rahmen nicht möglich ist. Es sei hier nur gesagt, daß in San Marino bis auf Römisches Recht zurückgegriffen wird, daß 
Maskat und Yemen islamisches Gewohnheitsrecht anwenden, während bei den Volksdemokratien China und Mongolei die StA-Prinzipien der marxistischen Staaten zu Grunde zu legen sind. So finden sich hier drei außerordentlich verschiedene Arten von Gewohnheitsrecht.

4. Die 13 Staaten, deren StA allein in der Verfassung geregelt ist, gehören meist dem britischen Rechtskreis und darin mit einer Ausnahme (Malediven) dem Commonwealth an. Es sind in Australien die winzige Insel Nauru, in Asien Malediven, Singapore und Malaysia und in Afrika sechs Staaten, nämlich Botswana, Lesotho, Nigeria, Swasiland, Sierra Leone, Uganda. Bei den meisten dieser Staaten gilt noch die durch britische Order in Council bei der Unabhängigkeit in Kraft gesetzte Verfassung, die in einem Kapitel die StA behandelt. Lediglich Malediven, Nauru, Singapore, Uganda erließen später eigene Verfassungen. Weitere ehemalige britische Kolonien, die bei der Unabhängigkeit die StA in der Verfassung geregelt hatten, erließen bald ein eigenes StAG und sind daher unten zu behandeln. Zu der hier behandelten Gruppe gehören jedoch noch drei Staaten, die mehr zufällig die StA zur Zeit nur in der Verfassung regeln: Burundi, Aquatorialguinea, Paraguay.

5. Die 16 Staaten, bei denen das StAR auf Verfassung und Gesetz verteilt ist, bilden wiederum einen in sich zusammengehörigen Rechtskreis, sie sind nämlich sämtlich in Lateinamerika belegen. Von den 20 ibero-amerikanischen Staaten haben nur vier eine hiervon abweichende Regelung: nämlich die drei Bundesstaaten Brasilien, Mexico, Argentinien (eigene StA-Gesetze neben der Verfassung), und Paraguay (ausschließlich Verfassung). Die 16 Staaten umfassen sieben südamerikanische (Bolivien, Chile, Columbien, Ecuador, Peru, Uruguay, Venezuela) und neun mittelamerikanische (Costarica, Cuba, Dominikanische Republik, Guatemala, Haiti, Honduras, Nicaragua, Panama, Salvador). Diese Staaten haben neben den häufig wechselnden Verfassungen eigene Einbürgerungsgesetze von größerem Bestand bzw. in Einzelfällen Ausländergesetze, die die Einbürgerung mit regeln.

6. Alle übrigen 102 Staaten, nämlich etwa $2 / 3$, regeln die StA durch besonderes Gesetz. Hierbei gibt es naturgemäß viele Variationen. Es gibt Staaten, die das StAR in zwei Gesetzen regeln, eines über die StA i. a. und eines über die Einbürgerung im besonderen (Laos, Philippinen, Vietnam-Nord). Manche ehem. britischen Staaten lassen neben dem StAG noch Vorschriften der Verfassung gelten, so daß diese Gruppen derjenigen unter Nr. 5 nahekommt (z. B. Guayana, Jamaica, Trinidad). Es gibt Staaten, die das StA-Recht innerhalb eines Gesetzes mit anderen Fragen verknüpfen, wie die USA mit dem Einwanderungsrecht und der Vatikan mit dem Ausländerrecht. Es gibt Staaten, in denen das StAR grundsätzlich in einem verwickelten System mehrerer Gesetze geregelt ist, wie in der CSSR nach ihrer Föderalisierung. Andere Staaten haben zwar ein einziges StAG, aber daneben Sonderregelungen, wie Deutschland, das im Grundgesetz und mehreren Sondergesetzen Regelungen neben das RuStAG von 1913 treten ließ. Es gibt Staaten, die ihr gesamtes Recht in einem Super-Code zusammengefaßt haben, wie Liberia: darin ist dann auch das StAR enthalten. Schließlich wechseln auch die Bezeichnungen dessen, was im weiteren Sinne hier als StAG bezeichnet wird: es kann sein Order, Ordinance, Ordannance, Dekret, Arrêté und im ehemaligen französischen Kolonialreich wird der materielle Inhalt dann oft als "Code de la Nationalité" bezeichnet: so in den 18 ehemaligen französischen Staaten Afrikas und in Vietnam-Süd.

In der folgenden Übersicht sind die 141 Staaten alphabetisch geordnet und jeweils Datum und Bezeichnung der Regelung angegeben. Dabei muß bemerkt werden, daß vor allem die älteren Regelungen meist Änderungen erlebt haben, die hier aufzuführen allerdings zu umfangreich würde.

Am Ende sind anhangweise noch die Regelungen derjenigen Protektorate aufgeführt, die eigene Vorschriften über die StA besitzen. Das sind vier britische Schutzstaaten in Asien (Bahrein, Brunei, Qatar, Tonga) und sechs britische Assoziierte 
Staaten in Westindien. Ferner gehört in gewisser Weise auch Erythräa noch hierzu. Alle anderen Protektorate (Bhutan, Sikkim, die sieben Trucial States, Brit. Solomon Islands Protectorate) besitzen keine geschriebene Regelung.

\section{Übersicht}

EBG: Einbürgerungsgesetz

StAG: Staatsangehörigkeitsgesetz

\begin{tabular}{|c|c|c|}
\hline Afghanistan & 8.11 .1936 & StAG \\
\hline Albanien & 7. 6.1954 & StA-Dekret \\
\hline Algerien & 27. 3.1963 & StAG \\
\hline Andorra & 17. 6.1939 & StA-Dekret \\
\hline Äquatorialguinea & 9.10 .1968 & Verfassung (Übergangsregel) \\
\hline Argentinien & 1. 10. 1869 & StAG \\
\hline Athiopien & 22. 7.1930 & StAG \\
\hline Australien & 21. 12. 1948 & StAG \\
\hline Barbados & 19. 8.1967 & StAG \\
\hline Belgien & 15. 5.1922 & StAG \\
\hline \multirow[t]{2}{*}{ Bolivien } & 2. 2. 1967 & Verfassung \\
\hline & 31. 8. 1951 & EBG \\
\hline Botswana & 20. 9. 1966 & Verfassung \\
\hline Brasilien & 18. 9. 1949 & StAG \\
\hline Bulgarien & 7.10. 1968 & StAG \\
\hline Burma & 8. 11.1948 & StAG \\
\hline Burundi & 16. 10.1962 & Verfassung \\
\hline Canada & 27. 6.1949 & StAG \\
\hline Ceylon & 21. 9.1948 & StAG \\
\hline \multirow{2}{*}{ Chile } & 18. 9.1925 & Verfassung \\
\hline & 16. 7.1941 & EB-Dekret \\
\hline \multicolumn{3}{|l|}{ China } \\
\hline China (Taiwan) & 5. 2. 1929 & StAG \\
\hline \multirow[t]{2}{*}{ Columbien } & 16. 2. 1945 & Verfassung \\
\hline & 3. 2. 1936 & EBG \\
\hline \multirow[t]{2}{*}{ Costarica } & 7.11. 1949 & Verfassung \\
\hline & 29. 4.1950 & EBG \\
\hline \multirow[t]{2}{*}{ Cuba } & 7. 2. 1959 & Verfassung \\
\hline & 4. 2. 1944 & EB-Dekret \\
\hline Cypern & 16. 8.1960 & Staatsvertrag \\
\hline Dänemark & 27. 5.1950 & StAG \\
\hline Dahome & 23. 6.1965 & StAG \\
\hline Deutschland (BRD) & 22. 7.1913 & StAG \\
\hline Deutschland (DDR) & 20. 2. 1967 & StAG \\
\hline \multirow[t]{2}{*}{ Dominikanische Republik } & 28.11 .1966 & Verfassung \\
\hline & 16. 4.1948 & EBG \\
\hline \multirow[t]{2}{*}{ Ecuador } & 29. 5.1967 & Verfassung \\
\hline & 20. 6.1950 & EB-Dekret \\
\hline Elfenbeinküste & 14. 12.1961 & StAG \\
\hline Finnland & 28. 6.1968 & StAG \\
\hline Frankreich & 19. 10.1945 & StAG \\
\hline Gabun & 2. 3. 1962 & StAG \\
\hline Gambia & 11. 2.1965 & StA-Ordinance \\
\hline Ghana & 7. 6.1961 & StAG \\
\hline
\end{tabular}




\begin{tabular}{|c|c|c|}
\hline Griechenland & 20. 9.1965 & StAG \\
\hline Großbritannien & 30. 7.1948 & StAG \\
\hline \multirow[t]{2}{*}{ Guatemala } & 15. 9.1965 & Verfassung \\
\hline & 25. 1. 1936 & Ausländer-Dekret \\
\hline Guayana & 31. 8.1967 & StAG \\
\hline Guinea & 1. 3.1960 & StAG \\
\hline \multirow[t]{2}{*}{ Haiti } & 29. 5.1964 & Verfassung \\
\hline & 3. 7.1941 & EBG \\
\hline \multirow[t]{2}{*}{ Honduras } & 3. 6.1965 & Verfassung \\
\hline & 1. 3.1946 & Ausländer-Gesetz \\
\hline Indien & 30. 12.1955 & StAG \\
\hline Indonesien & 29. 7. 1958 & StAG \\
\hline Irak & 30. 5.1963 & StAG \\
\hline Iran & 16. 2. 1935 & BGB \\
\hline Irland & 17. 7. 1956 & StAG \\
\hline Island & 23. 12. 1952 & StAG \\
\hline Israel & 1. 4.1952 & StAG \\
\hline Italien & 13. 6. 1912 & StAG \\
\hline Jamaica & 19. 12.1962 & StAG \\
\hline Japan & 4. 5.1950 & StAG \\
\hline Jordanien & 4. 2.1954 & StAG \\
\hline Jugoslawien & 17. 9. 1964 & StAG \\
\hline \multirow[t]{2}{*}{ Kambodscha } & 26. 7. 1915 & BGB \\
\hline & 27. 9.1959 & EBG \\
\hline Kamerun & 11. 6. 1968 & StAG \\
\hline Kenia & 9. 12. 1963 & StA-Ordinance \\
\hline Kongo (B) & 20. 6. 1961 & StAG \\
\hline Kongo (K) & 18. 9.1965 & StAG \\
\hline Korea (Nord) & 6.10 .1963 & StAG \\
\hline Korea (Süd) & 20. 12. 1948 & StAG \\
\hline Kuwait & 5. 12.1959 & StAG \\
\hline \multirow[t]{2}{*}{ Laos } & 6. 4. 1953 & StAG \\
\hline & 8. 3.1957 & EBG \\
\hline Lesotho & 20. 9.1966 & Verfassung \\
\hline Libanon & 19. 1.1925 & StA-Arrêté \\
\hline Liberia & 22. 3. 1956 & Code of laws \\
\hline Libyen & 18. 4.1954 & StAG \\
\hline Liechtenstein & 4. 1.1934 & StAG \\
\hline Luxemburg & 22. 2. 1968 & StAG \\
\hline Madagaskar & 22. 7.1960 & StAG \\
\hline Malawi & 14. 6.1966 & StAG \\
\hline Malaysia & 5. 8.1957 & Verfassung \\
\hline Malediven & 12. 1968 & Verfassung \\
\hline Mali & 3. 2.1962 & StAG \\
\hline Malta & 27. 8.1965 & StAG \\
\hline Marokko & 6. 9.1958 & StAG \\
\hline \multicolumn{3}{|c|}{ Maskat und Oman } \\
\hline Mauretanien & 12. 6.1961 & StAG \\
\hline Mauritius & 13. 12. 1968 & StAG \\
\hline Mexico & 5. 1.1934 & StAG \\
\hline \multirow[t]{2}{*}{ Monaco } & 12. 1.1818 & BGB \\
\hline & 18.11. 1952 & Gesetz über StA-Erwerb \\
\hline $\begin{array}{l}\text { Mongolei } \\
\text { Nauru }\end{array}$ & 29. 1. 1968 & Verfassung \\
\hline
\end{tabular}




\begin{tabular}{|c|c|c|}
\hline Nepal & 28. 2. 1964 & StAG \\
\hline Neuseeland & 6. 9.1948 & StAG \\
\hline \multirow[t]{2}{*}{ Nicaragua } & 1.11 .1950 & Verfassung \\
\hline & 8. 9.1894 & Ausländergesetz \\
\hline Niger & 12. 7.1961 & StAG \\
\hline Nigeria & 1. 10.1963 & Verfassung \\
\hline Niederlande & 12. 12.1892 & StAG \\
\hline Norwegen & 8. 12.1950 & StAG \\
\hline Obervolta & 1. 12.1961 & StAG \\
\hline Ơsterreich & 15. 7.1965 & StAG \\
\hline Pakistan & 13. 4. 1951 & StAG \\
\hline \multirow[t]{2}{*}{ Panama } & 1. 3.1946 & Verfassung \\
\hline & 6. 2. 1941 & $\mathrm{EBG}$ \\
\hline \multirow{3}{*}{$\begin{array}{l}\text { Peru } \\
\text { Parag }\end{array}$} & 26. 8.1967 & Verfassung \\
\hline & 9. 4.1933 & Verfassung \\
\hline & 13. 6. 1940 & EBG \\
\hline \multirow{2}{*}{ Philippinen } & 21.10 .1936 & StAG \\
\hline & 17. 6. 1939 & EBG \\
\hline Polen & 15. 2. 1962 & StAG \\
\hline Portugal & 29.7 .1959 & StAG \\
\hline Rhodesien & 30. 12.1963 & StAG \\
\hline Rumänien & 24. 1. 1952 & StA-Dekret \\
\hline Rwanda & 28. 9.1963 & StAG \\
\hline \multirow{2}{*}{ Salvador } & 8. 1.1962 & Verfassung \\
\hline & 27. 9.1886 & Ausländergesetz \\
\hline \multicolumn{3}{|l|}{ San Marino } \\
\hline Saudiarabien & 20.10. 1954 & StAG \\
\hline Schweden & 22. 6.1950 & StAG \\
\hline Schweiz & 29. 9.1952 & StAG \\
\hline Senegal & 7. 3. 1961 & StAG \\
\hline Sierra Leone & 14. 4. 1961 & Verfassung \\
\hline Singapore & 22. 12.1965 & Verfassung \\
\hline Somalia & 2. 12.1963 & StAG \\
\hline Sow jetunion & 19. 8.1938 & StAG \\
\hline Spanien & 26. 3. 1889 & BGB \\
\hline Südafrika & 29. 6.1949 & StAG \\
\hline Sudan & 25. 6. 1957 & StAG \\
\hline Südyemen & 11. 4.1968 & StAG \\
\hline Swasiland & 26. 8. 1968 & Verfassung \\
\hline Syrien & 31. 10. 1961 & StAG \\
\hline Tansania & 6. 12. 1961 & StA-Ordinance \\
\hline Thailand & 21. 7. 1964 & StAG \\
\hline Togo & 25. 7. 1961 & StAG \\
\hline Trinidad/Tobago & 30. 8.1962 & StAG \\
\hline Tschad & 14. 8.1962 & StAG \\
\hline \multirow[t]{4}{*}{ Tschechoslowakei } & 13. 7.1949 & StAG \\
\hline & 19. 12. 1968 & StAG \\
\hline & 29. 4. 1969 & StAG der Tschechischen SR \\
\hline & 28. 12. 1968 & StAG der Slowakischen SR \\
\hline Tunesien & 28. 2. 1963 & StAG \\
\hline Türkei & 11. 2.1964 & StAG \\
\hline Uganda & 8. 9.1967 & Verfassung \\
\hline Ungarn & 6. 6.1957 & StAG \\
\hline
\end{tabular}




\begin{tabular}{|c|c|c|}
\hline Uruguay & $\begin{array}{rr}\text { 24. } & 8.1966 \\
1 . & 2.1928\end{array}$ & $\begin{array}{l}\text { Verfassung } \\
\text { EBG }\end{array}$ \\
\hline Vatikan & 7. 6.1929 & StAG und Ausländergesetz \\
\hline Venezuela & $\begin{array}{ll}23 . & 1.1961 \\
18 . & 7.1955\end{array}$ & $\begin{array}{l}\text { Verfassung } \\
\text { EBG }\end{array}$ \\
\hline Ver. Arab. Rep. & 22. 6.1958 & StAG \\
\hline Ver. Staaten & 27. 6. 1952 & $\begin{array}{l}\text { StAG und } \\
\text { Einwanderungsgesetz }\end{array}$ \\
\hline Vietnam (Nord) & $\begin{array}{l}\text { 20. 10. } 1945 \\
\text { 25. 2. } 1946\end{array}$ & $\begin{array}{l}\text { Dekret betr. StA } \\
\text { Dekret betr. StA }\end{array}$ \\
\hline Vietnam (Süd) & 7.12. 1955 & StAG \\
\hline $\begin{array}{l}\text { West-Samoa } \\
\text { Yemen }\end{array}$ & 8. 9.1959 & StA-Ordinance \\
\hline $\begin{array}{l}\text { Zambia } \\
\text { Zentralafrika }\end{array}$ & $\begin{array}{l}\text { 19. } 10.1964 \\
27 . \quad 5.1961\end{array}$ & $\begin{array}{l}\text { StA-Ordinance } \\
\text { StAG }\end{array}$ \\
\hline
\end{tabular}

Anhang: Protektorate

\begin{tabular}{|c|c|c|}
\hline Antigua & 22. 2. 1967 & Verfassung \\
\hline Bahrein & 19. 9.1963 & StAG \\
\hline Brunei & 6. 12. 1961 & StAG \\
\hline Dominica & 22. 2. 1967 & Verfassung \\
\hline Erythräa & 2. 11.1953 & StAG \\
\hline Grenada & 22. 2. 1967 & Verfassung \\
\hline Qatar & 3. 4. 1961 & StAG \\
\hline St. Christopher-Nevis-Anguilla & 22. 2. 1967 & Verfassung \\
\hline St. Lucia & 22. 2. 1967 & Verfassung \\
\hline St. Vincent & 10. 1969 & Verfassung \\
\hline Tonga & 1947 & StAG \\
\hline
\end{tabular}

Eine berichtigte und ergänzte Fassung des Beitrages von Dr. Hellmuth Hecker über das Staatsangehörigkeitsrecht in Asien (VRU 1969, S. 329-367) kann bei der Redaktion kostenlos unter Beifügung des Rückportos angefordert werden. 